\title{
Diagnóstico dos Resíduos de Serviços de Saúde no INTERIOR DO RIO GRANDE do SUl
}

\section{Diagnosis of medical wastes in central Rio Grande do Sul State}

\author{
CARLOS ERNANDO DA SILVA \\ Mestre e doutor em Engenharia Química pela Universidade Estadual de Campinas. Professor Adjunto do Departamento \\ de Hidráulica e Saneamento - CT - UFSM
}

ALESSANDRO EDUARDO HOPPE

Acadêmico do curso de Engenharia Civil - UFSM. Bolsista de Iniciação Científica da FAPERGS

Recebido: 29/11/04 Aceito: 08/03/05

\section{RESUMO}

O presente trabalho tem como objetivo analisar e avaliar aspectos do gerenciamento dos resíduos de serviços de saúde (RSS) nos municípios pertencentes à bacia hidrográfica do rio Vacacaí, no estado do Rio Grande do Sul. Através de levantamento em campo, foram analisados os procedimentos adotados no manejo dos RSS gerados nos hospitais, postos de saúde e laboratórios clínicos. Todos os estabelecimentos de saúde apresentaram falhas nas várias fases da gestão de seus resíduos, não atendendo os princípios preconizados na Resolução CONAMA N. 283/2001. A taxa de geração de resíduos nos hospitais foi de $3,245 \mathrm{~kg} /$ leito.dia, sendo $17,6 \%$ referente aos resíduos do Grupo A. O total de resíduos gerados na área de estudo foi estimado em $182.640 \mathrm{~kg} / \mathrm{mês}$ (22,1\% do Grupo A).

PALAVRAS-CHAVE: Resíduos de serviços de saúde, gerenciamento de resíduos, geração de resíduos.

\begin{abstract}
The paper aims to analyze and evaluate the medical wastes management in the cities of Vacacai river basin in the State of Rio Grande do Sul, Brazil. Health care facilities (hospitals, health centers and clinical laboratories) were surveyed to provide information about the management, segregation, generation, storage and disposal of medical wastes. The results about management aspects indicate that practices in most healthcare facilities do not comply with the principles stated in Brazilian legislation (CONAMA Resolution N. 283/2001). Average generation rate of total waste in the hospitals was estimated to be 3,245 kg/bed.day (17.6\% from Group A). A total medical waste generated in the study area was estimated to be $182,640 \mathrm{~kg} /$ month $(22.1 \%$ from Group A).
\end{abstract}

KEYWORDS: Medical wastes, waste management, waste generation.

\section{INTRODUÇÃO}

Os Resíduos de Serviços de Saúde (RSS) são resíduos gerados por prestadores de assistência médica, odontológica, laboratorial, farmacêutica e instituiçôes de ensino e pesquisa médica relacionadas tanto à população humana quanta à veterinária. Os RSS, apesar de representarem uma pequena parcela em relação ao total de resíduos gerados em uma comunidade, são fontes potenciais de propagação de doenças e apresentam um risco adicional aos trabalhadores dos serviços de saúde e a comunidade em geral, quando gerenciados de forma inadequada.

A gestão brasileira dos RSS teve como marco a Resolução N. 5 do CONAMA (Brasil, 1993), sendo atribuídas responsabilidades específicas aos vários segmentos envolvidos como: geradores, autoridades sanitárias e ambientais. Esta resolução estabelece definições, classificação e procedimentos mínimos para o gerenciamento dos RSS, sendo os resíduos classificados segundo seus riscos:

- Grupo A: resíduos que apresentam risco à saúde pública e ao meio ambiente devido à presença de agentes biológicos;

Grupo B: resíduos que apresentam risco à saúde pública e ao meio ambiente devido as suas características físicas, químicas e físico-químicas:

- Grupo C: resíduos radioativos ou contaminados com radionuclídeos;

- Grupo D: São todos os demais resíduos que não se enquadram nos grupos descritos anteriormente.

A Resolução N. 283 do CONAMA (Brasil, 2001) complementa os procedimentos do gerenciamento, estabelecendo as diretrizes para o tratamento e disposição dos resíduos de serviços de saúde.

As diretrizes dos procedimentos técnicos para o gerenciamento dos RSS foram estabelecidas através da Resolução RDC N. 306 da ANVISA (Brasil, 2004), que buscou a harmonização dos princípios contemplados na Resolução N. 283 do CONAMA (Brasil, 2001).

De acordo com Moreal (1993) a taxa média brasileira de geração de RSS equivale a 2,63 Kg/leito.dia, sendo que cerca de 15-20\% deste total representam resíduos classificados no Grupo A (infectantes-biológicos). Entretanto, o manejo inadequado dos resíduos, pode promover a contaminação de toda a massa dos resíduos. Schneider et al (2002) desenvolveram um estudo sobre os resíduos gerados pelos consultórios odontológicos, apresentando uma geração total de $0,241 \mathrm{~kg} /$ dentista.dia, sendo que $0,180 \mathrm{~kg} /$ dentista.dia correspondente aos resíduos do Grupo A.

Atualmente, na bacia hidrográfica do rio Vacacaí são encontrados vários problemas devido à ação antrópica, resultando em impactos que promovem a degradação ambiental e prejuízos à saúde pública, estando os resíduos sólidos inseri- 
dos neste contexto. Todos os municípios que compõem a bacia apresentam uma deficiência marcante em relação à disposição final dos resíduos sólidos urbanos, sendo dispostos em lixões nas periferias dos centros urbanos. Quanto aos resíduos de serviços de saúde, não existem dados oficiais disponíveis abordando aspectos qualitativos e quantitativos. Silva et al (2002) apresentaram dados preliminares avaliando a situação dos RSS gerados nos hospitais dos municípios da bacia do Vacacaí, sendo observadas diversas falhas no sistema de gestão destes resíduos.

Este trabalho tem por objetivo analisar e avaliar a real situação dos resíduos dos serviços de saúde gerados pelos municípios da bacia hidrográfica do Rio Vacacaí, visando subsidiar informaçōes para o estabelecimento de diretrizes para o gerenciamento correto destes resíduos, levando em consideração normas e legislações vigentes.

\section{ÁREA DE ESTUDO}

O estudo foi realizado na bacia hidrográfica do rio Vacacaí, situada na região central do estado do Rio Grande do Sul (Figura 1). Esta bacia possui uma área de drenagem de $10.000 \mathrm{~km}^{2}$ e uma população total de 492.567 habitantes, distribuídos nos seus 11 municípios.

A partir de informaçōes obtidas junto à $4^{\mathrm{a} C o o r d e n a d o r i a ~ R e g i o n a l ~ d e ~ S a u ́ d e ~}$ do Rio Grande do Sul, Conselho Regional de Odontologia do Rio Grande do Sul e do trabalho de campo, foi realizado o cadastramento dos estabelecimentos prestadores de serviços de saúde no âmbito da área de estudo, os quais estão sintetizados na Tabela 1.

Ressalta-se que a cidade de Santa Maria é um centro de referência regional de saúde, tendo como principal suporte o Hospital Universitário da Universidade Federal de Santa Maria (HUFSM), que disponibiliza as diversas especialidades médicas no atendimento da população dos municípios da região.

De acordo com a legislação vigente, os governos municipais são responsáveis pela coleta e disposição final dos resíduos domésticos, de forma a atender os propósitos sanitários e ambientais. Entretanto, nenhum município possui um programa de gerenciamento dos resíduos e tão pouco um programa oficial para a reciclagem dos mesmos. A disposição dos resíduos é realizada de forma inadequada, a céu aberto (lixôes), na periferia dos centros urbanos. Ressalta-se a existência

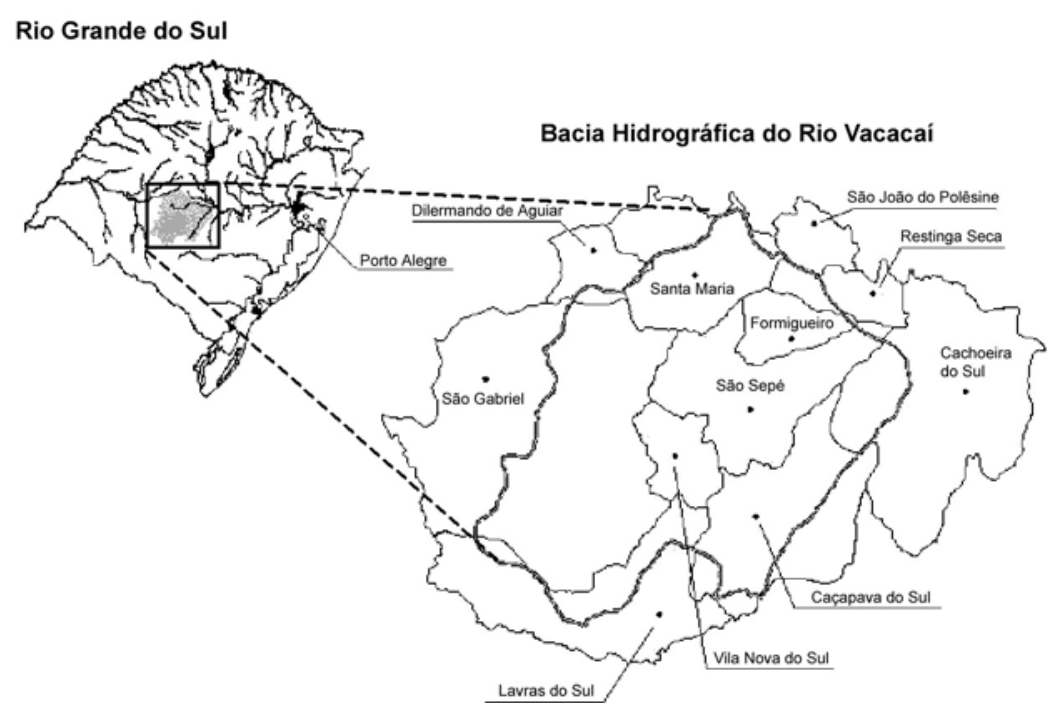

Figura I - Localização dos municípios da bacia do rio Vacacaí

Fonte: Modificado de Araújo (1998)

de algumas intervenções técnicas nestes "lixões", visando à transformação em aterro controlado. Entretanto, na prática, estas ações são descontinuadas e resultam apenas em melhorais pontuais em curtos intervalos de tempo. Não existem dados oficiais sobre os resíduos de serviços de saúde gerados. Atualmente, duas empresas privadas operam a coleta e disposição final destes resíduos gerados nos diversos estabelecimentos de serviços de saúde.

\section{METODOLOGIA}

O presente trabalho consiste em um estudo exploratório e descritivo da situação dos RSS nos municípios que compõem a bacia hidrográfica do Rio Vacacaí, através da aplicação de formulários e visitas de campo nas unidades de saúde da área de estudo.

Os tipos de unidades de saúde pesquisadas (hospitais, postos de saúde e

Tabela I - Número de estabelecimentos prestadores de serviços de saúde nos municípios da bacia hidrográfica do rio Vacacaí

\begin{tabular}{cccccc}
\hline Município & População* & $\begin{array}{c}\text { Hospitais/ } \\
\text { Leitos }\end{array}$ & $\begin{array}{c}\text { Postos de } \\
\text { saúde }\end{array}$ & $\begin{array}{c}\text { Laboratórios } \\
\text { clínicos }\end{array}$ & Dentistas \\
\hline Caçapava do Sul & 34.643 & $1 / 90$ & 1 & 2 & 21 \\
Cachoeira do Sul & 87.873 & $2 / 194$ & 5 & 2 & 82 \\
Dilermano de Aguiar & 3.200 & $0 / 0$ & - & - & - \\
Formigueiro & 7.598 & $1 / 23$ & 3 & - & 3 \\
Lavras do Sul & 8.109 & $1 / 32$ & 1 & 2 & 7 \\
Restinga Seca & 16.400 & $2 / 107$ & - & 2 & 10 \\
Santa Maria & 243.611 & $10 / 831$ & 28 & 8 & 590 \\
São Gabriel & 62.249 & $3 / 245$ & 9 & 4 & 45 \\
São Sepé & 24.621 & $1 / 57$ & - & 2 & 19 \\
Vila Nova do Sul & 4.263 & $0 / 0$ & 1 & - & 3 \\
Total Região & 492.567 & $21 / 1579$ & 48 & 22 & 780 \\
\hline *Fonte: IBGE (2002) & & &
\end{tabular}

*Fonte: IBGE (2002) 
laboratórios de análises clínicas) foram definidas por serem os principais geradores de RSS. As unidades de saúde foram identificadas através de consulta ao cadastro da $4^{a}$ Coordenadoria Regional de Saúde, em Santa Maria-RS.

Os formulários da pesquisa foram elaborados buscando englobar as principais informaçōes sobre os procedimentos gerenciais, operacionais e levantamento de dados qualitativos e quantitativos dos RSS gerados, abrangendo os aspectos relativos à segregação, acondicionamento, armazenamento interno e externo, transporte, tratamento e disposição final, adotando como referência a Resolução CONAMA N. 283 (Brasil, 2001).

A aplicação dos formulários foi realizada através do envio de correspondência ao responsável pela unidade de saúde, contendo o formulário e as instruções de preenchimento. O recebimento do formulário preenchido foi programado através de contato prévio com o responsável de cada unidade de saúde para visita de campo de um dos pesquisadores. Nesta etapa, buscou-se avaliar e confrontar os dados informados e a realidade observa$\mathrm{da}$, possibilitando um melhor esclarecimento para o completo e seguro preenchimento do formulário.

As informaçōes contidas nos formulários foram tabuladas e na estrutura de um banco de dados em planilha eletrônica para análise e avaliação da situação dos RSS, contemplando os aspectos legais no âmbito das legislações nas diferentes esferas de competência (federal, estadual e municipal). A partir dos dados quantitativos da geração de resíduos foi determinada a taxa de geração dos resíduos nos hospitais da região (kg/leito.dia), considerando uma densidade dos resíduos de $150 \mathrm{~kg} / \mathrm{m}^{3}$.

\section{RESULTADOS E DISCUSSÃO}

\section{Aspectos do gerenciamento}

A Tabela 2 apresenta os principais aspectos do gerenciamento dos RSS nos estabelecimentos prestadores de serviços de saúde, sendo demonstrados os percentuais dos estabelecimentos que responderam afirmativamente os vários itens avaliados.

Os resultados obtidos apontam que a maioria dos estabelecimentos prestadores de serviços de saúde não atende os procedimentos preconizados na Resolu- ção CONAMA N. 283/2001. Nos hospitais avaliados, apenas cerca de 30\% possuem Comissão de Resíduos de Serviços de Saúde (CRSS), com responsável técnico com formação específica; Plano de Gerenciamento dos Resíduos de Serviços de Saúde (PGRSS) e Programa de Treinamento e Capacitação dos Servidores (PTCS). Os laboratórios clínicos e os postos de saúde apresentaram um percentual de $10 \%$ e $0 \%$, respectivamente. A ausência destes suportes para o gerenciamento reflete negativamente nas diversas fases da gestão dos RSS, em especial, nas etapas finais de fluxo dos resíduos.

As vistorias técnicas realizadas pelos órgãos competentes, principalmente a vigilância sanitária, são mais freqüentes nos laboratórios clínicos, sendo que acima de $80 \%$ destes já foram vistoriados quantos aos aspectos dos RSS.

\section{Segregação dos resíduos}

Os dados apresentados na Figura 2 sugerem que os estabelecimentos de saúde demonstram uma prioridade na separação dos resíduos do Grupo A, isto é, perfuro-cortantes (RPC) e biológicosinfectantes (RBI).

Todos os hospitais promovem a segregação dos resíduos perfuro-cortantes, sendo que nos centros de saúde e laboratórios este percentual é de $98 \%$ e $95 \%$, respectivamente. Esses resíduos são segregados no ponto de geração e acondicionados em recipientes rígidos. Entretanto, cerca de $20 \%$ dos centros de saúde não promovem a segregação dos demais tipos de resíduos do Grupo A. Os resíduos biológicos-infectantes são acondicionados em sacos plásticos (geralmente de cor branca na maioria dos hospitais). De acordo com vários relatos na literatura, os perfuro-cortantes são os principais resíduos que estão associados à transmissão de doenças infecciosas. Isto não é surpresa, tendo em vista a capacidade intrínseca destes em romper a integridade da pele e introduzir agentes infecciosos no tecido. Assim, o alto nível de segregação dos perfuro-cortantes representa um fator importante da situação encontrada. Entretanto, foi relatado em várias ocasiōes durante as visitas aos estabelecimentos, que os resíduos segregados são misturados junto aos demais resíduos pelos servidores responsáveis pela coleta e transporte para estocagem externa. Portanto, enquanto a segurança dos servidores dos estabelecimentos de saúde é assegurada, em alguns casos, a situação do público em geral continua a mesma.

Apesar da exigência legal, os resíduos do Grupo B não têm recebido a atenção necessária em todos os estabelecimentos, como também pode ser observado através da Figura 2. Apenas cerca de $57 \%$ dos hospitais promovem a segregação destes resíduos, sendo uma problemática maior nos centros de saúde e laboratórios ( $42 \%$ e $14 \%$, respectivamente). Desde que os resíduos do Grupo B possuem um grande potencial de causar ou contribuir para a mortalidade ou apresentar um perigo substancial para a saúde humana e o meio ambiente, se gerenciado e disposto impropriamente, estes resultados observados descrevem um cenário preocupante da realidade detectada. A principal razão para a situação encontrada é devida principalmente ao nível de conhecimento insuficiente dos servidores dos estabelecimentos, acerca dos aspectos ambientais. O conhecimento básico dos servidores é centrado na área de ciências da saúde, o que justifica o alto desempenho na gestão dos resíduos do Grupo A.

Os resíduos recicláveis são separados em aproximadamente $70 \%, 40 \%$ e $50 \%$ dos hospitais, centros de saúde e laboratórios, respectivamente. Apesar dos valores relatados, nenhum estabelecimento correntemente está operando programas de reciclagem de resíduos não conta-

Tabela 2 - Percentual dos estabelecimentos que responderam afirmativamente aos aspectos do gerenciamento dos RSS

\begin{tabular}{cccc}
\hline $\begin{array}{c}\text { Aspectos do } \\
\text { gerenciamento }\end{array}$ & \multicolumn{3}{c}{ Estabelecimentos } \\
& Hospitais & Centros de saúde & Laboratórios clínicos \\
\hline CRSS & 28,6 & 0,0 & 13,6 \\
PGRSS & 28,6 & 4,2 & 9,1 \\
PTCS & 33,3 & 10,4 & 13,6 \\
Vistorias & 57,1 & 56,3 & 81,8 \\
\hline
\end{tabular}




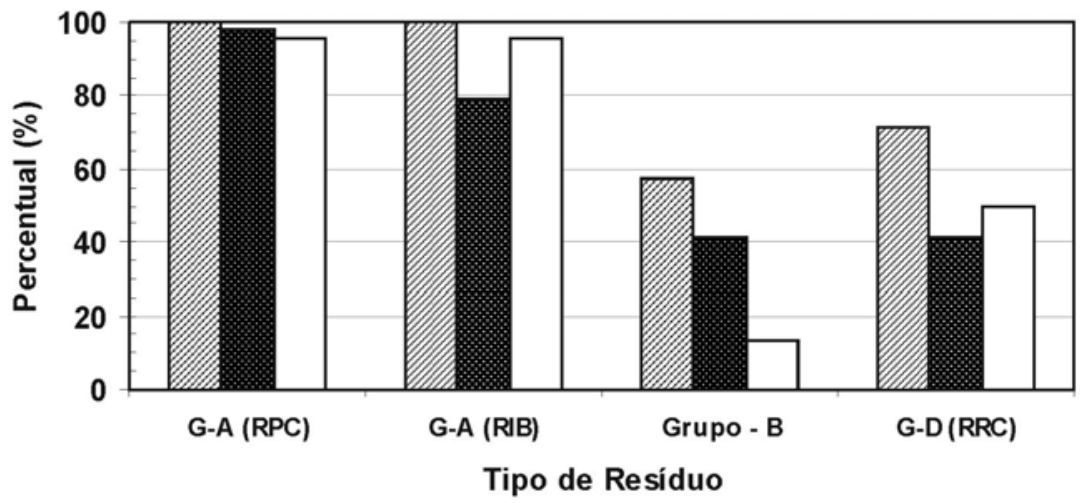

口 Hospitais 图Postos de Saúde $\square$ Laboratórios

Figura 2 - Percentual de segregação dos resíduos de serviços de saúde por unidade de saúde

minados, ou seja, papel, papelão, plástico, metal e vidro. Grande parte destes resíduos é coletada juntamente com os demais resíduos e dispostos em "lixões" nas periferias das cidades.

\section{Armazenamento dos resíduos}

As principais características das condições de armazenamento dos RSS são apresentadas na Tabela 3. Verifica-se que a maioria dos estabelecimentos apresenta deficiência quanto ao armazenamento interno e externo dos resíduos, principalmente em relação às condições físicas das áreas utilizadas. Os hospitais apresentam uma melhor estrutura de armazenamento, tendo em vista o maior volume de resíduos gerados. $\mathrm{O}$ transporte interno dos RSS, nos hospitais, é realizado em carros fechados sendo o equipamento de uso exclusivo para o serviço. Os centros de saúde e laboratórios, acondicionam os resíduos gerados e geralmente não promovem a estocagem interna e ou externa, sendo os mesmos disponibilizados diretamente para a coleta e disposição final.

\section{Aspectos quantitativos dos resíduos}

A quantificação dos resíduos foi o item que apresentou maior dificuldade para informação pelos responsáveis dos estabelecimentos que responderam os formulários. Nem todos os estabelecimentos foram capazes de quantificar os resíduos gerados. Os quantitativos foram apresentados em uma grande variedade de unidades (kg ou g ou litro/dia ou semana ou mês). Para propósitos de com- paração, todos os dados foram convertidos em base mássica (kg/mês), considerando a densidade dos resíduos de $150 \mathrm{~kg} / \mathrm{m}^{3}$.

A Tabela 4 apresenta o total de resíduos e os resíduos do Grupo A gerados nos hospitais. Considerando apenas os hospitais que apresentaram os quantitativos dos resíduos, foi estimada uma taxa de geração de 3,245 kg/leito.dia (total de resíduos) e 0,570 kg/leito.dia (resíduos do Grupo A). Os valores indicam que cerca de $18 \%$ dos resíduos gerados nos hospitais correspondem aos resíduos do Grupo A. Estas taxas estão de acordo com os valores obtidos por Moreal (1993). A Tabela 4 também apresenta a geração dos resíduos estimada por estas taxas. Verifica-se uma diferença significativa principalmente entre os valores estimados e aqueles informados pelos hospitais de pequeno porte. Estas constatações podem ser justificadas pelo baixo nível dos aspectos do gerenciamento discutidos anteriormente, onde apenas $30 \%$ dos hospitais possuem Comissão de Resíduos, Plano de Gerenciamento dos Resíduos e Programa de Capacitação e Treinamento dos Servidores.

Assumindo que o número total de leitos hospitalares na área em estudo é de 1579 e as taxas de geração de resíduos (total e do Grupo A) determinadas acima, verifica-se uma geração mensal total dos hospitais de 153.742 e $26.674 \mathrm{~kg} / \mathrm{mês}$ (resíduos totais e resíduos do Grupo A, respectivamente). A Tabela 5 sintetiza a geração média dos demais estabelecimentos. A quantidade de resíduos das clínicas odontológicas foi estimada considerando uma geração de $0,180 \mathrm{~kg} /$ dentista.dia, conforme sugerido por Schneider et al (2002). Verifica-se que a grande parte do total de resíduos gerados, cerca de $83 \%$, são provenientes dos hospitais. Entretanto, o conteúdo de resíduos do Grupo A é significativamente maior nos centros de saúde, laboratórios e consultórios odontológicos. $\mathrm{O}$ alto valor observado nos postos de saúde sugere uma baixa eficiência no gerenciamento, em especial na etapa de segregação dos resíduos, sendo que os resíduos comuns são, em vários estabelecimentos, acondicionados juntamente com os resíduos biológicosinfectantes do Grupo A.

\section{Coleta e disposição final dos resíduos}

A coleta dos RSS (Grupos A e B) na grande maioria dos estabelecimentos é realizada de forma diferenciada (coleta especial) por empresas privadas, através de contratos de prestação de serviços en-

Tabela 3 - Percentual das condições de armazenamento dos resíduos nos estabelecimentos

\begin{tabular}{ccccc}
\hline Armazenamento & $\begin{array}{c}\text { Condiçóes } \\
\text { físicas da área }\end{array}$ & \multicolumn{3}{c}{ Estabelecimentos } \\
& Hospitais & Postos de saúde & Laboratórios clínicos \\
\hline \multirow{2}{*}{ Interno } & Existente & 76,2 & 54,2 & 50,0 \\
& Uso exclusivo & 66,7 & 27,1 & 45,5 \\
\multirow{3}{*}{ Externo } & Identificada & 42,9 & 20,8 & 40,9 \\
& Existente & 85,7 & 37,5 & 40,9 \\
& Uso exclusivo & 85,7 & 29,2 & 45,5 \\
& Identificada & 61,9 & 29,2 & 22,7 \\
& Iluminada & 57,1 & 12,5 & 45,5 \\
& Cobertura & 71,4 & 29,2 & 50,0 \\
& Individualizada & 81,0 & 8,3 & 18,2 \\
\hline
\end{tabular}


Tabela 4 - Geração de resíduos nos hospitais - valores informados e estimados

\begin{tabular}{|c|c|c|c|c|c|}
\hline \multirow[t]{2}{*}{ Hospitais } & \multirow{2}{*}{$\begin{array}{l}\text { Leitos } \\
(\#)\end{array}$} & \multicolumn{2}{|c|}{ Informados } & \multicolumn{2}{|c|}{ Estimados } \\
\hline & & \multicolumn{4}{|c|}{ (Kg/mês) } \\
\hline 1 & 5 & 276,3 & 726,3 & 87,6 & 486,8 \\
\hline 2 & 8 & 1,1 & 136,1 & 140,2 & 778,9 \\
\hline 3 & 9 & 690,0 & $1.980,0$ & 157,7 & 876,3 \\
\hline 4 & 14 & $2.934,0$ & $4.959,0$ & 245,4 & $1.363,1$ \\
\hline 5 & 15 & 12,3 & 141,3 & 262,9 & $1.460,5$ \\
\hline 6 & 16 & 12,0 & 552,0 & 280,4 & $1.557,9$ \\
\hline 7 & 17 & 567,0 & $1.017,0$ & 297,9 & $1.655,2$ \\
\hline 8 & 23 & 300,0 & $1.800,0$ & 403,1 & $2.239,4$ \\
\hline 9 & 32 & 225,0 & 360,0 & 560,8 & $3.115,7$ \\
\hline 10 & 42 & - & - & 736,1 & $4.089,4$ \\
\hline 11 & 46 & 105,0 & $7.305,0$ & 806,2 & $4.478,9$ \\
\hline 12 & 52 & 151,8 & 303,6 & 911,4 & $5.063,1$ \\
\hline 13 & 55 & 16,7 & $2.716,7$ & 963,9 & $5.355,2$ \\
\hline 14 & 57 & 660,0 & 750,0 & 999,0 & $5.549,9$ \\
\hline 15 & 66 & 900,0 & $7.500,0$ & $1.156,7$ & $6.426,2$ \\
\hline 16 & 71 & $1.035,0$ & $5.310,0$ & $1.244,3$ & $6.913,0$ \\
\hline 17 & 90 & - & - & $1.577,3$ & $8.763,0$ \\
\hline 18 & 178 & $2.400,0$ & $4.800,0$ & $3.119,6$ & $17.331,3$ \\
\hline 19 & 220 & $3.420,0$ & $8.820,0$ & $3.855,7$ & $21.420,7$ \\
\hline 20 & 252 & $4.772,7$ & $76.412,7$ & $4.416,6$ & $24.536,4$ \\
\hline 21 & 311 & $6.300,0$ & $15.300,0$ & $5.450,6$ & $30.281,0$ \\
\hline Total & 1579 & $24.778,9$ & $140.889,7$ & $26.673,6$ & $153.742,0$ \\
\hline
\end{tabular}

Tabela 5 - Quantidade de resíduos gerados nos estabelecimentos de saúde

\begin{tabular}{cccc}
\hline Estabelecimentos & $\begin{array}{c}\text { Total } \\
(\mathrm{Kg} / \mathrm{mês})\end{array}$ & $\begin{array}{c}\text { Grupo A } \\
(\mathrm{kg} / \mathrm{mês})\end{array}$ & $\begin{array}{c}\text { Grupo A } \\
(\%)\end{array}$ \\
\hline Hospitais & 153.742 & 26.674 & 17,6 \\
Postos de saúde & 18.758 & 8.402 & 44,8 \\
Laboratórios clínicos & 6.380 & 2.449 & 38,4 \\
Dentistas & 3.760 & 2.808 & 74,7 \\
Total & 182.640 & 40.333 & 22,1 \\
\hline
\end{tabular}

tre as partes. Os resíduos do Grupo A são incinerados ou são depositados, sem tratamento, em valas sépticas. Os resíduos do Grupo B têm como destinação final o aterro de resíduos perigosos, no município de Estância Velha-RS.

$\mathrm{O}$ tratamento e a destinação final dos RSS é tema de grandes debates junto aos vários segmentos da sociedade dos municípios da bacia do Vacacaí, em especial os aspectos legais, na busca de uma solução consorciada entre os municípios. A base de grandes questionamentos era devido à Lei Orgânica dos municípios, que preconizava a obrigatoriedade da incineração dos RSS, atendendo as diretrizes da Portaria N. 53 do Ministério de Estado do Interior (BRASIL, 1979). Ressalta-se que esta obrigatoriedade foi revogada através da Resolução N. 5 do CONAMA (BRASIL, 1993), entretanto não houve a respectiva atualização das legislações no âmbito municipal. Outra importante restrição presente na Lei Municipal é referente à proibição de recebimento de resíduos de outros municípios.

A cidade de Santa Maria, sendo a maior geradora de resíduos e pela sua importância regional, promoveu em dezembro de 2001 a atualização de sua lei orgânica, possibilitando um melhor equacionamento dos problemas relativos ao tratamento e disposição dos RSS no âmbito regional. As modificações realizadas contemplaram a possibilidade de gestão dos resíduos de forma consorciada, entretanto, impondo a necessidade do tratamento dos RSS para a sua disposição final.

Frente à nova realidade regional, em outubro de 2002 uma empresa privada, situada na cidade de Santa Maria, obteve o licenciamento ambiental para operação de uma unidade de esterilização de RSS pelo processo de autoclavagem e posterior disposição final no aterro sanitário na Central de Resíduos Recreio, no município de Butiá - RS. Atualmente, o sistema de valas sépticas na cidade de Santa Maria, é utilizado para a disposição de carcaças de animais.

A região ainda possui uma unidade de incineração em funcionamento na cidade de Santa Maria. Tal sistema encontra-se em processo de regularização da operação, junto à Fundação Estadual do Meio Ambiente do Rio Grande do Sul FEPAM. Entretanto, acredita-se que a tendência será a desativação do mesmo, frente as grandes restriçōes técnicas quanto às emissốes atmosféricas estabelecidas na 
Resolução CONSEMA N. 009 (RS, 2000).

\section{CONCLUSÕES}

Verifica-se que esforços vêm sendo realizados junto às fontes geradoras, em especial nas etapas de segregação e acondicionamento dos resíduos do Grupo A. Entretanto, pouca atenção é dada aos demais tipos de resíduos, os quais geralmente são acondicionados sem uma perspectiva de reciclagem e são coletados pelo serviço de coleta pública municipal. $\mathrm{O}$ envolvimento da sociedade na discussão dos aspectos relativos aos RSS vem possibilitando o estabelecimento de diretrizes na solução da problemática apresentada, em especial para as etapas finais de fluxo dos resíduos. A realidade regional apresenta situação favorável quanto ao gerenciamento dos RSS, entretanto, distante de uma situação ideal. É importante ressaltar, que todos os municípios da bacia hidrográfica do rio Vacacaí promovem a disposição dos resíduos domésticos em "lixôes", localizados nas periferias dos centros urbanos, representando um grave problema sanitário, ambiental e social. A falta de controle adequado da disposição dos RSS pode agravar ainda mais este problema.

\section{AGRADECIMENTOS}

Os autores agradecem à Fundação de Apoio à Pesquisa do Estado do Rio Grande do Sul - FAPERGS pelo apoio recebido através de edital de auxílio à pesquisa e de bolsa de iniciação científica.

\section{REFERÊNCIAS}

ARAÚJO, A. Mapa de situação e localização da Bacia Hidrográfica do Rio Vacacaí (Mapa 01). In: Avaliação quali quantitativa das demandas e disponibilidades de água na bacia hidrográfica do rio vacacaí (bhrv). GOVERNO DO ESTADO DO RIO GRANDE DO SUL/SOPSH/ DRHS/FRHRS/CRH/RS. CD-ROM. 1998.

BRASIL. Ministério de Estado do Interior. Portaria $N^{\circ} 53$ de 01/03/1979. Brasília, 1979.

BRASIL. Resolução CONAMA No 05/1993. Define as normas mínimas para tratamento de resíduos sólidos oriundos de serviços de saúde, portos e aeroportos e terminais rodoviários e ferroviários. Diário Oficial da República Federativa do Brasil, 31 ago., Seção 1. Brasília, 1993.

BRASIL. Resolução CONAMA No 283/2001. Dispõe sobre o tratamento e a destinação final dos resíduos dos serviços de saúde. Diário Oficial da República Federativa do Brasil, 01 out., Seção 1. Brasília, 2001

BRASIL. Resolução ANVISA RDC N 306/2004, Dispóe sobre o Regulamento Técnico para o gerenciamento de resíduos de serviços de saúde. Diário Oficial da República Federativa do Brasil, 10 dez., Seção 1. Brasília, 2004.

INSTITUTO BRASILEIRO DE GEOGRAFIA E ESTATÍSTICA - IBGE - Senso 2000, Rio de Janeiro, 2002. Disponível em: ww.ibge.gov.br. Acesso em 19 dez. 2002.

MONREAL, J. Consideraciones sobre el Manejo de Resíduos de Hospitales en America Latina. In: SEMINÁRIO INTERNACIONAL SOBRE RESÍDUOS SÓLIDOS HOSPITALARES, 1993, Cascavel. Anais...Cascavel, PR: p. 2-24. 1993

RIO GRANDE DO SUL. Resolução CONSEMA No 009/2000. Licenciamento ambiental de sistemas de incineração de resíduos de serviços de saúde no estado do Rio Grande do Sul. Porto Alegre, 2000 .
SCHNEIDER, V.E., et al. Geração de Resíduos Sólidos de Serviços Odontológicos (RSSO) no Estado do Rio Grande do Sul com Base no Indice de Geração Per Capita - Subsídio para Implantação de Modelos de Gerenciamento. In: SEMINÁRIO NACIONAL DE RESÍDUOS SÓLIDOS, 6 , 2002. Gramado. Anais...Gramado, RS: Abes, CDROOM. 2002.

SILVA, C.E., et al. Resíduos de servicos de saúde gerados nos municípios da Bacia Hidrográfica do Rio Vacacaí - RS. In: SEMINÁRIO NACIONAL DE RESÍDUOS SÓLIDOS, 6, 2002, Gramado. Anais...Gramado, RS: Abes, CDROOM. 2002

Endereço para correspondência:

Carlos Ernando da Silva

Departamento de Hidráulica e

Saneamento - CT

Universidade Federal de Santa Maria

Faixa de Camobi, Km 9 - Campus

Universitário - Centro de

Tecnologia - HDS - Camobi

97 I 05-900 - Santa Maria - RS -

Brasil

Tel.: (55) 220-8786

Fax.: (55)220-8030

Email: ces@smail.ufsm.br 